\title{
Strategies for Prevention of Non-communicable Diseases in Seafarers and Fishermen: Lessons Learned
}

\author{
Olaf Jensen $^{1,2 *}$, George Charalambous ${ }^{3}$, Agnes Flores ${ }^{4}$, Fereshteh Baygi ${ }^{1,5}$, MLuisa Canals ${ }^{6}$ and Debbie Andrioti $^{1}$ \\ ${ }^{1}$ Centre of Maritime Health and Society, Institute of Public Health, University of Southern Denmark, Niels Bohrs vej 9, 6700 Esbjerg, Denmark \\ ${ }^{2}$ Faculty of Health, National University of Panama, Panama City, Panama \\ ${ }^{3}$ Hippokration Hospital, Athens, Greece \& Frederick University Cyprus, 1036 Nicosia, Cyprus \\ ${ }^{4}$ Caja Seguro Social, Vacamonte, Panamá \& Universidad Metropolitana de Educación Ciencia y Tecnología \\ ${ }^{5}$ Occupational Health Research Centre, Iran University of Medical Sciences, Tehran, Iran \\ ${ }^{6}$ University of Cadiz, Spain, Spanish Society of Maritime Medicine (SEMM) \& IMHA-Research
}

\begin{abstract}
Noncommunicable diseases (NCDs) are responsible for almost twothirds of all deaths globally. Of the 57 million global deaths in 2008, 36 million, or $63 \%$, were due to NCDs, principally cardiovascular diseases, diabetes, cancers and chronic respiratory diseases. Mental health is now also seen as part of the chronic noncommunicable diseases [1]. These diseases share more or less the same risk factors (tobacco use, unhealthy diet, physical inactivity and the harmful use of alcohol and harmful mental health risks factors). As the impact of NCDs increases and as population ages, annual NCD deaths is projected to continue to rise worldwide [2]. In our research institute, we focus on seafarers and fishermen but the examples could easily be applied for other industries as well. Our aim is to inspire other health professionals to do systematic review studies of relevance for their specific target populations and to plan proved effective strategies.
\end{abstract}

Seafaring employees face more difficult working and living conditions than their peers on land. Their health is affected by the environment in which they live, often coupled with long working hours that contribute to eating more carbohydrate food and less physical activity in a working environment with high demands.

Study examples: In a seafaring population $45-66$ years of age $77 \%$ was overweight with a BMI $>25$ while only $60 \%$ in the same age group was overweight in 2010 [3]. Among the 50-59 years old seafarers $67 \%$ had hypertension and 30\% had pre-hypertension. BP $\geq 140 / 90 \mathrm{~mm}$ $\mathrm{Hg}$ was recorded as hypertension and between 120-140/80-90 as prehypertension [4]. For comparison, 33\% in the age group 55-64 of Danish workers had abnormal high HBP (Home Blood Pressure) and $25 \%$ had either white coat hypertension $(\mathrm{WCH})$ or masked hypertension $(\mathrm{MH})[5]$.

\section{Do Primary Prevention Programs Work?}

A review of the literature of the effectiveness of worksite nutrition and physical activity programs comprised 48 studies in all. Most of the studies combined informational and behavioural strategies to influence diet and physical activity; fewer studies modified the work environment (e.g., cafeteria, exercise facilities) to promote healthy choices. This review found that worksite nutrition and physical activity programs achieve modest improvements in employees' weight status at the 6-12 months follow-up [7]. A Cochrane review based on 57 studies with multiple primary risk factor interventions had little or no impact on the risk of coronary heart disease mortality or morbidity. The effects of attempting behaviour change in the general population do not appear to be effective [8]. Swinburne et al. concluded in the Lancet that increases in obesity in almost all countries seem to be driven mainly by changes in the global food system. Unlike other major causes of preventable death and disability, such as tobacco use, there are no examples of population trials in which the obesity epidemic has been reversed by public health measures [9].

\section{Publication History:}

Received: July 03, 2018

Accepted: August 01, 2018

Published: August 03, 2018

\section{Keywords:}

General health checks, Primary prevention, Routine health tjeck, Secondary prevention

Statins are the most widely prescribed, cholesterol-lowering drugs in the world. However, according to many studies there is only limited evidence showed that primary prevention with statins may be cost effective and improve patient quality of life. Caution should be taken in prescribing statins for primary prevention among people at low cardiovascular risk [10].

A structural health promotion intervention was implemented in two Danish shipping companies. (healthy cooking courses for ship cooks and improvement of fitness facilities) as well as health education interventions (smoking cessation courses, individual exercise guidance and extra health check-ups) at the maritime workplace. Significant changes were identified for levels of fitness, daily sugar intake and metabolic syndrome. The findings suggest that a multicomponent health promotion intervention program has the potential to achieve change in seafarers' health behaviour and health parameters [11].

\section{Do Routine Health Check-ups Work in Primary Prevention?}

Study examples: A Cochrane review with 11940 deaths did not find an effect of general health checks on total mortality or on mortality due to cardiovascular diseases [12].

Another community based, individually tailored intervention programme with screening for risk of ischaemic heart disease and repeated lifestyle intervention over five years had no effect on ischaemic heart disease, stroke, or mortality at the population level after 10 years [13].

*Corresponding Author: Dr. Olaf Jensen, Centre of Maritime Health and Society, Institute of Public Health, University of Southern Denmark, Niles Bohrs vej 9,6700, Esbjerg, Denmark; Email: ocj@health.sdu.dk

Citation: Jensen O, Charalambous G, Flores A, Baygi F, Canals M, et al. (2018) Strategies for Prevention of Non-communicable Diseases in Seafarers and Fishermen: Lessons Learned. Int J Community Fam Med 3: 142. https://doi. org/10.15344/2456-3498/2018/142

Copyright: () 2018 Jensen et al. This is an open-access article distributed under the terms of the Creative Commons Attribution License, which permits unrestricted use, distribution, and reproduction in any medium, provided the original author and source are credited. 
Citation: Jensen O, Charalambous G, Flores A, Baygi F, Canals M, et al. (2018) Strategies for Prevention of Non-communicable Diseases in Seafarers and Fishermen: Lessons Learned. Int J Community Fam Med 3: 142. https://doi.org/10.15344/2456-3498/2018/142

Page 2 of 2

Krogsbøll et al. identified 16 randomised trials which had compared a group of adults offered general health checks to a group not offered health checks. Results were available from 14 trials, including 182,880 participants. Nine trials studied the risk of death and included 155,899 participants and 11,940 deaths. There was no effect on the risk of death nor on the risk of death due to cardiovascular diseases or cancer [12].

A sample of 524 Danish seafarers who underwent medical fit-forduty examination by seamen's doctors were tracked and re-examined after 2 years. At baseline, all participants received general advice regarding lifestyle issues. Twenty-four percent of the seafarers were found with Metabolic Syndrome and they were additionally given specific advice regarding treatment. After the two years of follow-up, smoking and alcohol consumption was not reduced. In spite of the intervention, the prevalence of Metabolic Syndrome increased in this group of seafarers. This study indicates the limitations of individual health promotion and the need for corporate actions [14].

\section{Do Secondary Prevention Programs Help?}

Secondary prevention is defined as health risk prevention among persons with clinically apparent diseases. Study examples: Fortyfour studies were included in a systematic review of studies with patients from the US Diabetes Prevention Program (DPP) lifestyle modification program. The authors concluded that the Diabetes Prevention Program study showed weight loss in high-risk adults lowered diabetes incidence and cardiovascular disease risk [15]. In another review study with data from 74 trials it was concluded that intensive diet and physical activity behavioural counselling in persons with risk factors for cardiovascular disease resulted in consistent improvements across various important intermediate health outcomes up to 2 years [16].

\section{Conclusions}

Based on this limited literature review and the few examples, primary prevention studies with personal diet and physical activity behavioural counselling do not seem to work. Routine health checkups and advice in primary prevention do not seem to help either. Secondary prevention with intensive diet and physical activity behavioural counselling in persons with high risk factors for diabetes and cardiovascular disease seems to be effective.

\section{Recommendations}

Comprehensive structural preventive programs with the needed legislations and regulations are recommended by the WHO and other International Health Institutions [17,18]. Such programs should include a combination of fiscal policies, legislation, changes to the environments. Raising awareness of health risks by promoting healthier diets physical activity that include healthy mental health environments. The tasks for the universities and health professionals are monitoring and research, building up current knowledge about the health risks in work and leisure time to be used for the training. Clinicians, patient organisations, workers organisations and research institutions work together to provide the necessary knowledge and training programs. The aim is to establish comprehensive programs for changes for healthy occupational environments, healthy diet and physical activities in healthy mental cultures. Leaders and all teams in the industries must be trained and inspired by the public health professionals to establish a public health culture that shows the way forward with the use of best practices and strategies.

\section{Competing Interests}

The authors declare that no competing interests is present.

\section{References}

1. O'Neil A, Jacka FN, Quirk SE, Cocker F, Taylor CB, et al. (2015) A shared framework for the common mental disorders and Non-Communicable Disease: key considerations for disease prevention and control. BMC Psychiatry 15:15.

2. WHO (2018) Overview Preventing chronic diseases: a vital investment. WHO.

3. Hoeyer JL, Hansen HL (2005) Obesity among Danish seafarers. Int Marit Health 56: 48-55.

4. Tu M, Jepsen JR (2016) Hypertension among Danish seafarers. Int Marit Health 67: 196-204.

5. Hoffmann-Petersen N, Lauritzen T, Bech JN, Pedersen EB (2016) High Prevalence of Hypertension in a Danish Population Telemedical Home Measurement of Blood Pressure in Citizens Aged 55-64 Years in Holstebro County. Am J Hypertens 29: 439-447.

6. Harris MF, Parker SM, Litt J, van Driel M, Russell G, et al. (2017) An Australian general practice based strategy to improve chronic disease prevention, and its impact on patient reported outcomes: evaluation of the preventive evidence into practice cluster randomised controlled trial. BMC Health Serv Res 17: 637.

7. Anderson LM, Quinn TA, Glanz K, Ramirez G, Kahwati LC, et al. (2009) The effectiveness of worksite nutrition and physical activity interventions for controlling employee overweight and obesity: a systematic review. Am J Prev Med 37: 340-357.

8. Ebrahim S, Taylor F, Ward K, Beswick A, Burke M, et al. (2011) Multiple risk factor interventions for primary prevention of coronary heart disease. Cochrane Database Syst Rev 19: CD001561.

9. Swinburn BA, Sacks G, Hall KD, McPherson K, Finegood DT, et al (2011) The global obesity pandemic: shaped by global drivers and local environments. Lancet 378: 804-814.

10. Taylor F, Ward K, Moore TH, Burke M, Davey Smith G, et al. (2011) Statins for the primary prevention of cardiovascular disease. Cochrane Database Syst Rev 31: CD004816.

11. Hjarnoe L, Leppin A (2013) Health promotion in the Danish maritime setting: challenges and possibilities for changing lifestyle behavior and health among seafarers. BMC Public Health 13: 1165.

12. Krogsbø|l LT, Jørgensen KJ, Gøtzsche PC (2013) General health checks in adults for reducing morbidity and mortality from disease. JAMA 309: 24892490.

13. Jørgensen T, Jacobsen RK, Toft U, Aadahl M, Glümer C, et al. (2014) Effect of screening and lifestyle counselling on incidence of ischaemic heart disease in general population: Inter99 randomised trial. BMJ 348: g3617.

14. Jepsen JR, Rasmussen HB (2016) The metabolic syndrome among Danish seafarers: a follow-up study. Int Marit Health 67: 129-136.

15. Mudaliar U, Zabetian A, Goodman M, Echouffo-Tcheugui JB, Albright AL et al. (2016) Cardiometabolic Risk Factor Changes Observed in Diabetes Prevention Programs in US Settings: A Systematic Review and Metaanalysis. PLoS Med 13: e1002095.

16. Lin JS, O'Connor E, Evans CV, Senger CA, Rowland MG, et al. (2014) Behavioral counseling to promote a healthy lifestyle in persons with cardiovascular risk factors: a systematic review for the U.S. Preventive Services Task Force. Ann Intern Med 161: 568-578.

17. WHO (2018) 2008-2013 Action plan for the global strategy for the prevention and control of noncommunicable diseases. WHO

18. Saxena S, Funk M, Chisholm D (2013) World health assembly adopts comprehensive mental health action plan 2013-2020. The Lancet 381: 1970-1971. 